\title{
Research Article \\ Dynamic Analysis of a Model for Spruce Budworm Populations with Delay
}

\author{
Ahmadjan Muhammadhaji (iD) and Azhar Halik \\ College of Mathematics and System Sciences, Xinjiang University, Urumqi 830046, China \\ Correspondence should be addressed to Ahmadjan Muhammadhaji; ahmatjanam@aliyun.com
}

Received 13 August 2021; Accepted 21 September 2021; Published 29 September 2021

Academic Editor: Gisele Mophou

Copyright (c) 2021 Ahmadjan Muhammadhaji and Azhar Halik. This is an open access article distributed under the Creative Commons Attribution License, which permits unrestricted use, distribution, and reproduction in any medium, provided the original work is properly cited.

\begin{abstract}
A class of delayed spruce budworm population model is considered. Compared with previous studies, both autonomous and nonautonomous delayed spruce budworm population models are considered. By using the inequality techniques, continuation theorem, and the construction of suitable Lyapunov functional, we establish a set of easily verifiable sufficient conditions on the permanence, existence, and global attractivity of positive periodic solutions for the considered system. Finally, an example and its numerical simulation are given to illustrate our main results.
\end{abstract}

\section{Introduction}

As is well known, since the spruce budworm population site model [1] has been proposed and was accepted by numerous scholars, during the last decade, spruce budworm population models have been extensively investigated both in theory and applications, such as for protection of spruce trees and development of a strategy for spruce budworm population control [1-13]. For example, in [2], the authors considered the following standard structured partial differential model of the budworm population:

$$
\frac{\partial N}{\partial x}(x, b)+\frac{\partial N}{\partial b}(x, b)=-\bar{E}(b) N(x, b)-P_{b} N(x, b),
$$

where $N(x, b)$ is the size distribution of the budworm population and $\bar{E}(b)$ and $P_{b}$ are the mortality rate and the predation rate.

On the other hand, in mathematical modeling of real world problems, the growth rate of a natural species will not often respond immediately to changes in its own population or that of an interacting species but will rather do so after a time lag [14]. Recently, some delayed mathematical models have been proposed in the study of spruce budworm population models $[2,11-13]$, and some research results were obtained. For example, in [11], the authors further ana- lyzed system (1) and proposed the following delayed spruce budworm population model:

$$
\frac{d m(t)}{d t}=-D m(t)-p(m(t)) m(t)+e^{-\tilde{d} \tilde{\tau}} b(m(t-\tilde{\tau})),
$$

where $m(t)=\int_{\tilde{\tau}}^{\infty} N(t, a) d a$ is the mature population density at time $t, \tilde{\tau}$ is the maturation time, $D$ is the average mortality rate of the mature budworms, $\tilde{d}$ is the average death rate of the immature population, $p=p(m(t))$ is a predation rate function for the matured population, and $b=b(m(t))$ is the birth function. In [11], the authors nondimensionalize system (2) and obtained the following delayed nondimensional spruce budworm population model:

$$
\dot{y}(t)=-A y(t)-\frac{y^{2}(t)}{1+y^{2}(t)}+B y(t-\tau) e^{-C y(t-\tau)},
$$

where $A=D \gamma / \beta$ is related to the death of the matured population, $B=q_{1} \gamma e^{-\tilde{d} \tau} / \beta$ and $C=\alpha_{1} \gamma$ are related to birth and survival of the immature population, $\tau=\tilde{\tau} \beta / \gamma$ is a time delay, and $q_{1}$ is the birth rate-related parameter, and the meaning of other parameters of model (3) is given in [11]. The authors in [11] have studied system (3) on the equilibrium analysis, oscillation, and periodic outbreaks. After that, 
the authors in [13] have studied the dynamic behaviors of system (3) and obtained some sufficient conditions on the local stability of the positive equilibrium and Hopf bifurcation occurrence.

On the other hand, the autonomous systems (2) and (3) irrespective of the environmental changes have some limitations in mathematical modeling of ecological systems. Moreover, to the best of our knowledge, no study has been conducted to date for dynamics on the nonautonomous population model with stage structure for spruce budworm. Hence, based on the above models and analysis, in this paper, we study the following delayed nonautonomous population model with stage structure for spruce budworm:

$$
\dot{y}(t)=-A(t) y(t)-\frac{y^{2}(t)}{1+y^{2}(t)}+B(t) y(t-\tau) e^{-C(t) y(t-\tau)} .
$$

The interaction between the spruce budworm and the forests is one of the important themes in mathematical ecology due to the protection of spruce and balsam fir trees. In addition, the main problems in spruce budworm population models are the boundedness, permanence, extinction of the population, and the existence of the periodic solution and global attractivity of the system. Hence, in this paper, our main purpose is to establish some sufficient conditions on the above mentioned dynamical behaviors for systems (3) and (4).

\section{Preliminaries}

In system (4), $y(t)$ denote the density of the spruce budworm population and $\tau$ is a time delay. In this study, for system (4), we introduce the following basic assumption:

$\left(H_{1}\right) \tau>0$ and $A(t), B(t), C(t)$ are all continuously positive $\omega$-periodic functions on $[0, \omega]$

The following is the initial condition for system (4):

$$
y(t)=\phi(t) \quad \text { for all } t \in[-\tau, 0)
$$

where $\phi(t)$ is nonnegative continuous functions defined on $[-\tau, 0)$ and satisfying $\phi(0)>0$.

For a $\omega$-periodic continuous function $f(t)$ defined on $[0, \omega]$, we define $f^{L}=\inf _{t \in[0, \omega]}\{f(t)\}$ and $f^{M}=\sup _{t \in[0, \omega]}\{f$ $(t)\}$.

Now, we present some useful lemmas.

Lemma 1 (see [15]). If $\alpha>0, \beta>0$ and $\dot{u}(t) \geq(\leq) \beta-\alpha u(t)$, when $t \geq 0$ and $u(0)>0$, we have

$$
u(t) \geq(\leq) \frac{\beta}{\alpha}\left[1+\left(\frac{\alpha u(0)}{\beta}-1\right) e^{-\alpha t}\right]
$$

Lemma 2 (see [16]). Consider the following delay differential equation:

$$
\dot{x}(t)=a x(t-\tau)-b x(t)
$$

where $a, b, \tau$ are all positive constants and $x(t)>0$ for $t \epsilon$ $[-\tau, 0]$, we have the following:

(1) If $a<b$, then $\lim _{t \rightarrow+\infty} x(t)=0$

(2) If $a>b$, then $\lim _{t \longrightarrow+\infty} x(t)=+\infty$

Lemma 3 (continuation theorem [17]). Let $X$ and $Z$ be two Banach spaces. Suppose that $L: D(L) \subset X \longrightarrow Z$ is a Fredholm operator with index zero and $\tilde{N}: X \longrightarrow Z$ is L-compact on $\bar{\Omega}$, where $\Omega$ is an open subset of $X$. Moreover, assume that all the following conditions are satisfied:

(a) $L x \neq \lambda \tilde{N} x$, for all $x \in \partial \Omega \cap D(L), \lambda \in(0,1)$

(b) $\tilde{N} x \notin I m L$, for all $x \in \partial \Omega \cap \operatorname{Ker} L$

(c) The Brouwer degree $\operatorname{deg}\{Q \tilde{N}, \Omega \cap \operatorname{Ker} L, 0\} \neq 0$

Then, equation $L x=\tilde{N} x$ has at least one solution in Dom $L \cap \bar{\Omega}$.

\section{Boundedness, Extinction, and Periodic Solution}

Theorem 4. Assume that the assumption $\left(H_{1}\right)$ holds, then for any positive solution $y(t)$ of system (4), there exists a constant $M$ such that

$$
y(t) \leq M
$$

where

$$
M=\frac{B^{M}}{e C^{L} A^{L}} .
$$

Proof. From the equation of system (4) and for $t>\tau$, we have

$$
\begin{aligned}
\dot{y}(t) \leq & B^{M} y(t-\tau) e^{-C^{L} y(t-\tau)}-A^{L} y(t)=\frac{B^{M}}{C^{L}} C^{L} y(t-\tau) \\
& \cdot e^{-C^{L} y(t-\tau)}-A^{L} y(t)
\end{aligned}
$$

Then applying the following inequality $[16,18]$,

$$
\max _{x \geq 0} x e^{-x} \leq \frac{1}{e}
$$

we have

$$
\dot{y}(t) \leq \frac{B^{M}}{e C^{L}}-A^{L} y(t) .
$$

By Lemma 1, we get

$$
y(t) \leq \frac{B^{M}}{e C^{L} A^{L}}\left[1+\left(\frac{e C^{L} A^{L} y(0)}{B^{M}}-1\right) e^{-A^{L} t}\right],
$$


which yields

$$
\lim _{t \rightarrow+\infty} y(t) \leq \frac{B^{M}}{e C^{L} A^{L}} .
$$

Finally, there exists $T_{0}>0$ such that

$$
y(t) \leq \frac{B^{M}}{e C^{L} A^{L}}:=M
$$

for $t>T_{0}$.

For system (3), we have the following result.

Corollary 5. Assume that $A>0, B>0, C>0$, then for any positive solution $y(t)$ of system (3), there exists a constant $M^{\prime}>0$ such that

$$
y(t) \leq M^{\prime}
$$

where

$$
M^{\prime}=\frac{B}{e C A} .
$$

Theorem 6. Spruce budworm species $Y$ of system (4) is extinct if $\left(H_{1}\right)$ holds and $B^{M}<A^{L}$.

Proof. From the equation of system (4) and for $t>\tau$, we have

$$
\dot{y}(t) \leq B^{M} y(t-\tau)-A^{L} y(t)
$$

Note the following equation:

$$
\dot{x}(t)=B^{M} x(t-\tau)-A^{L} x(t) .
$$

By Lemma 2, we derive

$$
\lim _{t \longrightarrow+\infty} x(t)=0
$$

By comparison, there exists $T_{3}>0$ such that $y(t) \longrightarrow 0$ for $t \geq T_{3}$.

For system (3), we have the following result.

Corollary 7. Assume that $A>0, B>0, C>0$, and $B<A$. Then species $Y$ of autonomous system (3) is extinct, that is,

$$
\lim _{t \longrightarrow+\infty} y(t)=0
$$

Theorem 8. Suppose that assumption $\left(H_{1}\right)$ holds, then system (4) has at least one positive $\omega$-periodic solution.
Proof. Let $y(t)=\exp \{u(t)\}$, then system (4) can be rewritten as

$$
\begin{aligned}
\dot{u}(t)= & -A(t)-\frac{\exp \{u(t)\}}{1+\exp \{2 u(t)\}} \\
& +\frac{B(t) \exp \{u(t-\tau)\}}{\exp \{C(t) \exp \{u(t-\tau)\}+u(t)\}}:=\Gamma(u, t) .
\end{aligned}
$$

Let, $X=Z=\{u \in \mathscr{C}(R, R): u(t+\omega)=u(t)\}$ be Banach spaces equipped with the norm $\|\cdot\|$, where $\|u\|=$ $\max _{t \in[0, \omega]}|u(t)|$. Thus, we have for any $u \in X$, it is easy to see that $\Gamma(u, \cdot) \in \mathscr{C}(\mathbb{R}, \mathbb{R}) \omega$-periodic. Let

$$
\begin{gathered}
L: D(L)=\left\{u \in X: u \in \mathscr{C}^{1}(\mathbb{R}, \mathbb{R})\right\} \ni u \mapsto \dot{u}=\frac{d u}{d t} \in Z, \\
P: X \ni u \mapsto \frac{1}{\omega} \int_{0}^{\omega} u(s) d s \in X, \\
Q: Z \ni z \mapsto \frac{1}{\omega} \int_{0}^{\omega} z(s) d s \in Z, \\
\tilde{N}: X \ni u \mapsto \Gamma(u, \cdot) \in Z .
\end{gathered}
$$

We easily see

$$
\begin{aligned}
\operatorname{Im} L & =\left\{u \mid u \in Z, \int_{0}^{\omega} u(s) d s=0\right\} \\
\operatorname{Ker} L & =\mathbb{R} \\
\operatorname{Im} P & =\operatorname{Ker} L \\
\operatorname{Im} L & =\operatorname{Ker} Q=\operatorname{Im}(I-Q) .
\end{aligned}
$$

Therefore, operator $L$ is a Fredholm mapping of index zero. Furthermore, denoting by $L_{P}^{-1}: \operatorname{Im} L \longrightarrow D(L) \cap$ ker $P$ the inverse of $\left.L\right|_{D(L) \cap \text { Ker } P}$, we have

$$
L_{P}^{-1} v(t)=\int_{0}^{t} v(s) d s-\frac{1}{\omega} \int_{0}^{\omega} \int_{0}^{t} v(s) d s d t
$$

Thus, we have

$$
\begin{aligned}
Q \tilde{N} u= & \frac{1}{\omega} \int_{0}^{\omega} \tilde{N} u(t) d t=\frac{1}{\omega} \int_{0}^{\omega}\left[-A(t)-\frac{\exp \{u(t)\}}{1+\exp \{2 u(t)\}}\right. \\
& \left.+\frac{B(t) \exp \{u(t-\tau)\}}{\exp \{C(t) \exp \{u(t-\tau)\}+u(t)\}}\right] d t,
\end{aligned}
$$




$$
\begin{aligned}
& L_{P}^{-1}(I-Q) \tilde{N} u=\left(1-\frac{t}{\omega}\right) \int_{0}^{t} \tilde{N} u(s) d s-\frac{1}{\omega} \int_{0}^{\omega} \int_{0}^{t} \tilde{N} u(s) d s d t \\
& +\frac{1}{\omega} \int_{0}^{\omega} \int_{0}^{t} Q \tilde{N} u(s) d s d t,
\end{aligned}
$$

and $(I-Q) \tilde{N} u \in \operatorname{ImL}$, for all $u \in X$.

As in [18], we can easily show that for any open bounded set $\Omega \in X, \tilde{N}$ is $L$-compact on $\bar{\Omega}$. For the operator equation $L x=\lambda \tilde{N} x, \lambda \in(0,1)$, we have

$$
\dot{u}(t)=\lambda \Gamma(u, t) .
$$

Suppose that $u \in X$ is a solution of (28), for some $\lambda \in(0$ ,1), then there exist $\xi, \eta \in[0, \omega]$ such that

$$
\begin{gathered}
u(\xi)=\max _{t \in[0, \omega]} u(t), \\
u(\eta)=\min _{t \in[0, \omega]} u(t), \\
\dot{u}(\xi)=\dot{u}(\eta)=0 .
\end{gathered}
$$

It follows from (28) and (29) that

$$
\begin{aligned}
0= & \dot{u}(\xi)=\lambda\left[-A(\xi)-\frac{\exp \{u(\xi)\}}{1+\exp \{2 u(\xi)\}}\right. \\
& \left.+\frac{B(\xi) \exp \{u(\xi-\tau)\}}{\exp \{C(\xi) \exp \{u(\xi-\tau)\}+u(\xi)\}}\right] \\
0= & \dot{u}(\eta)=\lambda\left[-A(\eta)-\frac{\exp \{u(\eta)\}}{1+\exp \{2 u(\eta)\}}\right. \\
& \left.+\frac{B(\xi) \exp \{u(\eta-\tau)\}}{\exp \{C(\xi) \exp \{u(\eta-\tau)\}+u(\eta)\}}\right] .
\end{aligned}
$$

From (30), we obtain

$$
\begin{aligned}
A(\xi) \leq & \frac{B(\xi) u(\xi) \exp \{-u(\xi)\}}{u(\xi) C(\xi)} \\
& \cdot C(\xi) \exp \{u(\xi-\tau)\} \exp \{-C(\xi) \exp \{u(\xi-\tau)\}\} .
\end{aligned}
$$

By (11) and (32), we further have

$$
u(\xi) \leq \frac{B(\xi)}{e^{2} A(\xi) C(\xi)} \leq \frac{B^{M}}{A^{L} C^{L}}:=G_{1} .
$$

Next, from (31), we further obtain

$$
u(\eta) \geq \ln \left(\frac{A^{L}}{B^{M}}\right):=G_{2} .
$$

Let $G>\max \left\{G_{1},\left|G_{2}\right|\right\}$ be a fixed constant and define $\Omega=\{u \in X:\|u\|<G\}$. Then (33) and (34) imply that there is no $\lambda \in(0,1)$ and $u \in \partial \Omega$ such that $L u=\lambda \tilde{N} u$.
When $u \in \partial \Omega \cap \operatorname{Ker} L=\partial \Omega \cap R, u= \pm G$. Then we claim

$$
\begin{gathered}
Q \tilde{N}(-G)>0, \\
Q \tilde{N}(G)<0 .
\end{gathered}
$$

Otherwise, if $Q \tilde{N}(-G) \leq 0$, it follows from (26) that

$$
\begin{aligned}
B^{M} \exp \{-G\} & \geq \frac{1}{\omega} \int_{0}^{\omega} B(t) \exp \{-G\} \\
\cdot d t & \geq \frac{1}{\omega} \int_{0}^{\omega}\left[A(t)+\frac{\exp \{-G\}}{1+\exp \{-2 G\}}\right] d t \geq A^{L},
\end{aligned}
$$

which yields

$$
-G>\ln \left(\frac{A^{L}}{B^{M}}\right):=G_{2}
$$

This is a contradiction and implies that $Q \tilde{N}(-G)>0$. If $Q \tilde{N}(G) \geq 0$, from (11) and (26), it follows that

$$
A^{L} \leq \frac{1}{\omega} \int_{0}^{\omega} A(t) d t \leq \frac{1}{\omega} \int_{0}^{\omega}\left[\frac{B(t) \exp \{G\}}{\exp \{C(t) \exp \{G\}+G\}}\right] d t \leq \frac{B^{M}}{e^{2} G C^{L}},
$$

which implies that

$$
G \leq \frac{B^{M}}{A^{L} C^{L}}:=G_{1}
$$

a contradiction to the choice of $G$. Thus, $Q \tilde{N}(G)<0$. Define continuous function $G(u, \mu)$ by setting

$$
\begin{aligned}
G(x, \mu)= & -(1-\mu) u+\mu \frac{1}{\omega} \int_{0}^{\omega}\left[-A(t)-\frac{\exp \{u\}}{1+\exp \{2 u\}}\right. \\
& \left.+\frac{B(t)}{\exp \{C(t) \exp \{u\}\}}\right] d t .
\end{aligned}
$$

It follows from (35) that

$$
G(u, \mu) \neq 0 \quad \text { for all } u \in \partial \Omega \cap \operatorname{Ker} L .
$$

Using the homotopy invariance theorem, we obtain

$$
\begin{aligned}
& \operatorname{deg}\{Q \tilde{N}, \Omega \cap \operatorname{Ker} L, 0\}=\operatorname{deg}\left\{\frac { 1 } { \omega } \int _ { 0 } ^ { \omega } \left[-A(t)-\frac{\exp \{u\}}{1+\exp \{2 u\}}\right.\right. \\
& \left.\left.+\frac{B(t)}{\exp \{C(t) \exp \{u\}\}}\right] d t, \Omega \cap \operatorname{Ker} L, 0\right\} \\
& =\operatorname{deg}\{-u, \Omega \cap \operatorname{Ker} L, 0\} \neq 0 .
\end{aligned}
$$

It then follows from the continuation theorem (Lemma 3) that $L u=\tilde{N} u$ has a solution

$$
u^{*} \in \operatorname{Dom} L \cap \bar{\Omega},
$$


which is an $\omega$-periodic solution to equation (22). Therefore, $y^{*}(t)=\exp \left\{u^{*}(t)\right\}$ is a positive $\omega$-periodic solution of (4).

\section{Global Attractivity and Permanence}

Theorem 9. Suppose that $\left(H_{1}\right)$ holds and $A^{L}>B^{M} / C^{L} e^{2}$. Then system (4) has a positive w-periodic solution which is globally attractive.

Proof. From Theorem 8, we can obtain that system (4) has a positive periodic solution. Let $y^{*}(t)$ be a positive periodic solution of system (4) and $y(t)$ be any positive solution of system (4). From the boundedness of positive periodic solution $y^{*}(t)$ and Theorem 4 , we can choose positive constants $m$ and $M$ such that $m \leq y^{*}(t) \leq M$ and $y(t)<M$. Let

$$
\begin{aligned}
V(t)= & \left|y^{*}(t)-y(t)\right|+\int_{t-\tau}^{t} B(s+\tau) \mid y^{*}(s) e^{-C(s+\tau) y^{*}(s)} \\
& -y(s) e^{-C(s+\tau) y(s)} \mid d s .
\end{aligned}
$$

Calculating the upper right derivative of $V(t)$ along system (4), we have

$$
\begin{aligned}
D^{+} V(t)= & \operatorname{sign}\left(y^{*}(t)-y(t)\right)\left[-A(t)\left(y^{*}(t)-y(t)\right)-\left(\frac{\left(y^{*}\right)^{2}(t)}{1+\left(y^{*}\right)^{2}(t)}-\frac{y^{2}(t)}{1+y^{2}(t)}\right)\right. \\
& \left.-B(t)\left(y^{*}(t-\tau) e^{-C(t) y^{*}(t-\tau)}-y(t-\tau) e^{-C(t)(t-\tau)}\right)\right] \\
& +B(t+\tau)\left|y^{*}(t) e^{-C(t+\tau) y^{*}(t)}-y(t) e^{-C(t+\tau) y(t)}\right| \\
& -B(t)\left|y^{*}(t-\tau) e^{-C(t) y^{*}(t-\tau)}-y(t-\tau) e^{-C(t) y(t-\tau)}\right| \\
= & \operatorname{sign}\left(y^{*}(t)-y(t)\right)\left[-A(t)\left(y^{*}(t)-y(t)\right)-\left(\frac{\left(y^{*}(t)+y(t)\right)\left(y^{*}(t)-y(t)\right)}{\left(1+\left(y^{*}\right)^{2}(t)\right)\left(1+y^{2}(t)\right)}\right)\right. \\
& \left.-B(t)\left(y^{*}(t-\tau) e^{-C(t) y^{*}(t-\tau)}-y(t-\tau) e^{-C(t) y(t-\tau)}\right)\right] \\
& +B(t+\tau)\left|y^{*}(t) e^{-C(t+\tau) y^{*}(t)}-y(t) e^{-C(t+\tau) y(t)}\right|-B(t) \mid y^{*}(t-\tau) e^{-C(t) y^{*}(t-\tau)} \\
& -y(t-\tau) e^{-C(t) y(t-\tau)}\left|\leq-A^{L}\right| y^{*}(t)-y(t)\left|-\frac{m}{\left(1+M^{2}\right)^{2}}\right| y^{*}(t)-y(t) \mid \\
& +B^{M} \mid y^{*}(t) e^{-C(t+\tau) y^{*}(t)}-y(t) e^{-C(t+\tau) y(t) \mid} .
\end{aligned}
$$

Since

$$
\left|p e^{-p}-q e^{-q}\right|=\left|\frac{1-(p+\theta(q-p))}{e^{p+\theta(q-p)}}\right||p-q| \leq \frac{1}{e^{2}}|p-q|
$$

where $p, q \in[k,+\infty), 0<\theta<1$.

From (45) and (46), we have

$$
\begin{aligned}
D^{+} V(t) \leq & -A^{L}\left|y^{*}(t)-y(t)\right|-\frac{m}{\left(1+M^{2}\right)^{2}}\left|y^{*}(t)-y(t)\right| \\
& +\frac{B^{M}}{C^{L} e^{2}}\left|y^{*}(t)-y(t)\right| .
\end{aligned}
$$

Then we have

$$
D^{+} V(t) \leq-H\left|y^{*}(t)-y(t)\right|
$$

where $H=A^{L}+m /\left(1+M^{2}\right)^{2}-B^{M} / C^{L} e^{2}$.

Integrating from 0 to $t$ on both sides of (48) produces

$$
V(t)+H \int_{0}^{t}\left|y^{*}(s)-y(s)\right| d s \leq V(0) .
$$

Hence, $V(t)$ bounded on $[0, \infty)$ and we have

$$
\int_{0}^{t}\left|y^{*}(s)-y(s)\right| d s<\frac{V(0)}{H} .
$$

By the definition of $V(t)$ and (49), we have

$$
\left|y^{*}(t)-y(t)\right| \leq V(t) \leq V(0)
$$

Therefore, from boundedness of $y^{*}(t)$ and (49), we have

$$
m \exp \{-V(0)\} \leq y(t) \leq M \exp \{V(0)\}
$$

From the boundedness of $y^{*}(t)$ and (52), we have $y(t)$ bounded for $t \geq 0$. Then, we can obtain that $\left(y^{*}(t)-y(t)\right)$, and their derivatives remain bounded on $[0, \infty)$. As a consequence, $\left|y^{*}(t)-y(t)\right|$ is uniformly continuous on $[0, \infty)$. By Barbalat's lemma, it follows that

$$
\lim _{t \rightarrow \infty}\left|y^{*}(t)-y(t)\right|=0
$$

Hence,

$$
\lim _{t \rightarrow \infty}\left(y^{*}(t)-y(t)\right)=0 .
$$

Corollary 10. Suppose that $\left(H_{1}\right)$ holds and $A^{L}>B^{M} / C^{L} e^{2}$; then, system (4) is permanent.

Proof. From the global attractivity of system (4) and inequality (52), we can obtain the permanence of system (4).

Corollary 11. Assume that $A>0, B>0, C>0$ and $A>B / C e^{2}$, then system (3) is globally attractive.

\section{Example and Numerical Simulation}

Example 1. Investigate the following system:

$$
\begin{aligned}
\dot{y}(t)= & -(0.5+0.05 \cos (t)) y(t)-\frac{y^{2}(t)}{1+y^{2}(t)} \\
& +(2.155+0.1 \cos (t)) y(t-1) e^{-(0.81+0.01 \cos (t)) y(t-1)} .
\end{aligned}
$$




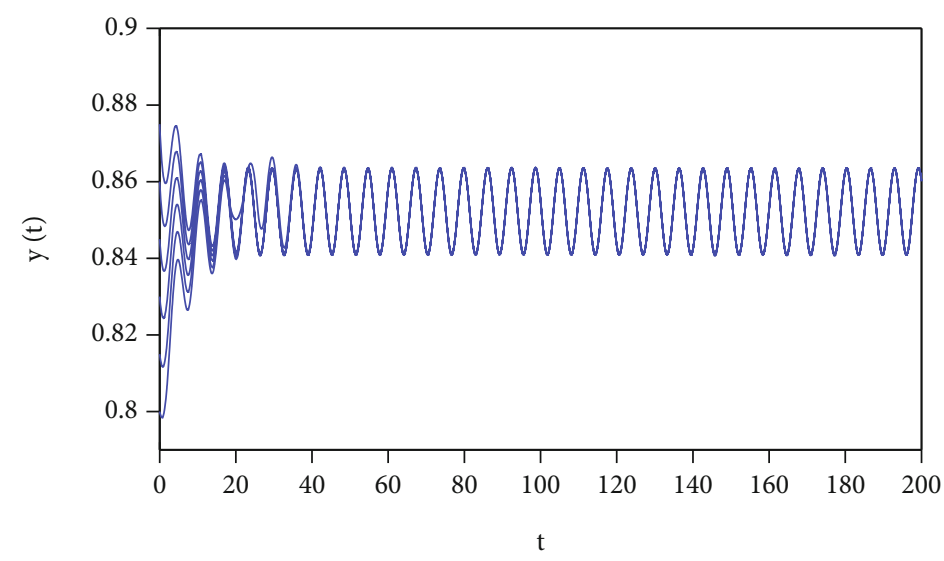

Figure 1: Dynamics of system (55). Here, we take different initial values.

Directly from calculation, we get

$$
A^{L}-\frac{B^{M}}{C^{L} e^{2}} \approx 0.0685
$$

Obviously, system (55) satisfies the conditions of Theorem 9. Hence, system (55) has a globally attractive positive periodic solution and is permanent. The existence and global attractivity of positive periodic solution and permanence of system (55) are shown in Figure 1.

\section{Conclusion}

In this paper, a class of delayed nonautonomous population model with stage structure for spruce budworm is proposed, and based on the inequality techniques, the comparison method, continuation theorem, and the construction of suitable Lyapunov functional, some new sufficient conditions on the boundedness, permanence, extinction, periodic solution, and global attractivity are obtained. Simultaneously, we also study system (3) and have obtained several conditions on the boundedness, extinction, and global attractivity of system (3). Because we extend systems (2) and (3) to system (4), we also obtained some sufficient conditions on the above-mentioned dynamical behaviors for the considered system. Hence, system (4) and the results obtained in this study can be seen as the supplements and extensions of previously known studies [11-13].

\section{Data Availability}

No data were used to support this study.

\section{Conflicts of Interest}

The authors declare that they have no competing interests.

\section{Acknowledgments}

This project is supported by the Natural Science Foundation of Xinjiang (Grant No. 2021D01C067) and the National Natural Science Foundation of China (Grant Nos. 11662020 and 11861063).

\section{References}

[1] D. D. Jones, "The budworm site model," Pest Management, Proceedings of an International Conference, , pp. 91-155, Pergamon Press, Oxford, 1976.

[2] W. H. S. Joseph, J. Wu, and X. Zou, "Structured population on two patches: modeling dispersal and delay," Journal of Mathematical Biology, vol. 43, no. 1, pp. 37-51, 2001.

[3] J. D. Murray, Mathematical Biology I: An Introduction, Springer, New York, 2002.

[4] R. I. Alfaro, J. Berg, and J. Axelson, "Periodicity of western spruce budworm in Southern British Columbia, Canada," Forest Ecology and Management, vol. 315, pp. 72$79,2014$.

[5] V. G. Nealis, R. Turnquist, B. Morin, R. I. Graham, and C. J. Lucarottic, "Baculoviruses in populations of western spruce budworm," Journal of Invertebrate Pathology, vol. 127, pp. 7680, 2015.

[6] D. C. Hassell, D. J. Allwright, and A. C. Fowler, "A mathematical analysis of Jones's site model for spruce budworm infestations," Journal of Mathematical Biology, vol. 38, no. 5, pp. 377421, 1999.

[7] M. Singh, A. Easton, and I. Kozlova, "A numerical study of the spruce budworm reaction diffusion equation with hostile boundaries," Natura L Resource Modeling, vol. 13, no. 4, pp. 535-549, 2000.

[8] B. Lubor, K. Alois, and P. Lucie, "Canard solutions and travelling waves in the spruce budworm population model," Applied Mathematics and Computation, vol. 183, no. 2, pp. 1039-1051, 2006.

[9] L. Wang, "Entire solutions of the spruce budworm model," Advances in Difference Equations, vol. 2018, no. 1, 2018.

[10] A. Rasmussen, J. Wyller, and J. O. Vik, "Relaxation oscillations in spruce-budworm interactions," Nonlinear Analysis: Real World Applications, vol. 12, no. 1, pp. 304-319, 2011.

[11] N. K. Vaidya and J. Wu, "Modeling spruce budworm population revisited: impact of physiological structure on outbreak control," Bulletin of Mathematical Biology, vol. 70, no. 3, pp. 769-784, 2008.

[12] X. Xu and J. Wei, "Bifurcation analysis of a spruce budworm model with diffusion and physiological structures," Journal of Differential Equations, vol. 262, no. 10, pp. 5206-5230, 2017. 
[13] J. Cao, J. Tan, and P. Wang, "Hopf bifurcation analysis of a model for spruce budworm populations with delays," Applied Mathematics and Mechanics, vol. 40, no. 3, pp. 332-342, 2019.

[14] A. Muhammadhaji, Z. Teng, and L. Zhang, "Permanence in general non-autonomous Lotka-Volterra predator-prey systems with distributed delays and impulses," Journal of Biological Systems, vol. 21, no. 2, pp. 1350012-1350028, 2013.

[15] A. Muhammadhaji, Z. Teng, and X. Abdurahman, "Permanence and extinction analysis for a delayed, ratio-dependent cooperative system with stage structure," Afrika Matematika, vol. 25, no. 4, pp. 897-909, 2014.

[16] A. Muhammadhaji and A. Halik, "Dynamics in a nonautonomous Nicholson-type delay system," Journal of Mathematics, vol. 2021, Article ID 6696453, 7 pages, 2021.

[17] R. Games and J. Mawhin, "Coincide degree and nonlinear differential equations," in Lecture Notes in Mathematics, vol. 1.568, Springer, Berlin, 1977.

[18] W. Wang, "Positive periodic solutions of delayed Nicholson's blowflies models with a nonlinear density-dependent mortality term," Applied Mathematical Modelling, vol. 36, no. 10, pp. 4708-4713, 2012. 